\title{
0 Custo Direto da fratura de fêmur por quedas em pessoas idosas: análise no Setor Privado de Saúde na cidade de Brasília, 2009
}

Direct Cost of femoral fracture from falls in elderly: analysis of the Private Section Health in the city of Brasilia, 2009

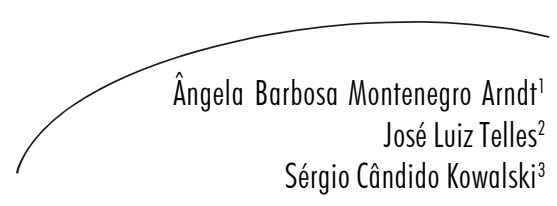

Resumo

Objetivo: estimar o custo direto dos recursos médico-hospitalares utilizados no tratamento cirúrgico da fratura de fêmur decorrente de quedas durante a hospitalização e pós-operatório até dois meses após a lesão. Método: estudo transversal, descritivo e retrospectivo em pacientes de ambos os sexos, com 60 anos ou mais de idade, no período de janeiro de 2008 a junho de 2009. Os dados foram extraídos das contas hospitalares de um convênio credenciado à rede hospitalar privada da cidade de Brasília. Resultados: a frequência de fratura por quedas para o sexo feminino foi $76,2 \%(\mathrm{n}=16)$, e no masculino, $23,8 \%(\mathrm{n}=5)$, sendo que $66,7 \%(n=14)$ das lesões resultaram em fratura de fêmur proximal. A média de permanência hospitalar das cirurgias foi de 7,1 dias e 2,7 dias em UTI. Houve 16 pacientes com fratura de fêmur e a média do tratamento cirúrgico foi $\mathrm{R} \$ 39.160,75$, totalizando $\mathrm{R} \$ 626.572,06$. O custo direto dos recursos médicohospitalares durante a internação ficou entre $\mathrm{R} \$ 8.293,55$ e $\mathrm{R} \$ 139.837,50$; já no pós-operatório, corresponderam a 5,7\% do custo total da internação. Conclusão: o custo cirúrgico da fratura de fêmur é considerável para os convênios do sistema de saúde suplementar e confirmou a vulnerabilidade do sexo feminino para as fraturas de quadril. As maiores despesas foram com a prótese, material e medicamentos, e variaram em função do tipo de cirurgia, período de hospitalização e faixa etária.

\section{Abstract}

Objective: to assess the direct cost of surgical treatment of femoral fracture resulting from falls during the hospitalization and surgery up to two months after injury. Methods: cross-sectional, descriptive and retrospective study conducted in patients of both sexes, aged 60 years or older, from January 2008 to June 2009. Data were

\footnotetext{
Programa de Pós-graduação em Gerontologia, Universidade Católica de Brasília. Brasília, DF, Brasil.

2 Escola Nacional de Saúde Pública/Fundação Oswaldo Cruz. Rio de Janeiro, RJ, Brasil.

3 Núcleo de Pesquisa em Economia da Saúde, Universidade Federal de São Paulo. São Paulo, SP, Brasil.
}

Palavras-chave: Saúde da Terceira idade. Setor Privado. Centro Cirúrgico hospitalar. Custos e análise de custo. Fratura de fêmur. Acidentes por quedas. Serviços de saúde para idosos. 
extracted from hospital bills of an agreement accredited to private hospital network in the city of Brasilia. Results: the frequency of femoral fracture for females was $76.2 \%(\mathrm{n}=16)$ and $23.8 \%(\mathrm{n}=5)$ for males. The average hospital stay for surgery was 7.1 days and 2.7 days in ICU. There were 16 patients with femoral fractures. The average expenditures for surgery was $\mathrm{R} \$ 39.160,74$ resulting from the all $R \$ 626,572.06$. The direct cost of hospitalization was between $R \$ 8.293 .55$ and $\mathrm{R} \$ 139,837.50$, but the hospital medical resources used in the postoperative period accounted for $5.7 \%$ of the direct cost of hospitalization. Conclusions: the cost per femoral fracture surgery is significant to the Brazilian private health system and females are more vulnerable than males. The highest costs were with the prosthesis, material and medicines, and they varied according to the type of surgery, hospital stay and age.
Key words: Health of the Elderly . Private Sector. Surgery Department Hospital Costs and cost analysis. Femoral fractures. Accidental falls. Health services for the elderly.

\section{INTRODUÇÃO}

O Brasil está vivenciando rápido processo de envelhecimento populacional. Os avanços da medicina e as melhorias nas condições gerais de vida da população repercutem na expectativa média de vida do brasileiro, que em 1940 era de 45,5 anos de idade, passando para 72,7 anos em 2008, ou seja, mais 27,2 anos de vida. Segundo a projeção do IBGE, o país continuará galgando anos na vida média de sua população, alcançando em 2050 o patamar de 81,29 anos. $^{1}$

Segundo o Departamento de Censo dos Estados Unidos, em poucos anos, logo após 2010, a proporção de pessoas mais idosas (acima de 75 anos) começará a crescer rapidamente na maioria dos países desenvolvidos e em muitos países em desenvolvimento. ${ }^{2}$

Envelhecer com qualidade de vida está diretamente relacionado com a ausência de incapacidade funcional, ainda que na velhice se experimente a presença de uma ou mais doenças crônicas. Quando falamos em "envelhecer”, as principais preocupações que surgem dizem respeito a como serão vividos os anos ganhos com o aumento da expectativa de vida e quem cuidará dessa população crescente. ${ }^{3}$

Diante do atual conceito gerontológico do “envelhecer saudável”, está o paradigma da capacidade funcional, ou seja, o idoso ao longo de seu processo do envelhecimento deveria manter suas habilidades físicas e mentais necessárias para uma vida independente e autônoma. Dentro dessa perspectiva, envelhecer saudável é o resultado da interação multidimensional entre saúde física, saúde mental, independência na vida diária, integração social, suporte familiar e independência econômica. ${ }^{4}$

No decorrer do ciclo de vida, qualquer pessoa está exposta a diversas situações que poderão levála à perda da autonomia e da independência, sendo uma delas a queda. ${ }^{5} \mathrm{~A}$ marcha depende $\mathrm{da}$ capacidade de vários órgãos, especificamente os dos sistemas neurológico, músculo-esquelético e cardiovascular. A principal tarefa motora durante a marcha envolve o controle do corpo e está integrado às atividades de vida diária (AVDs). ${ }^{6}$

A queda pode ser definida como o deslocamento não-intencional do corpo para um nível inferior à posição inicial com incapacidade de correção em tempo hábil, determinado por circunstâncias multifatoriais e comprometendo a estabilidade. ${ }^{7}$ A perda do equilíbrio postural pode estar relacionada à insuficiência súbita dos mecanismos neurais e osteomusculares envolvidos na manutenção da postura. ${ }^{6}$

Os fatores associados às causas das quedas estão classificados como intrínsecos, ou seja, decorrentes de alterações fisiológicas relacionadas ao envelhecimento, a doenças ou efeitos causados pelo uso de fármacos; e como extrínsecos, fatores que dependem de circunstâncias sociais e ambientais. As quedas podem impactar a vida do idoso de forma irreversível, não apenas pelas 
fraturas delas decorrentes, mas também pelas consequências advindas da reabilitação póscirúrgica, dentre as quais se encontram morbidades, mortalidade, deterioração funcional, hospitalização, institucionalização, aumento no consumo de medicamentos e de serviços de saúde. ${ }^{8}$ Pesquisa realizada no serviço de emergência dos Estados Unidos verificou que as quedas são eventos frequentes causadores de lesões, constituindo a principal etiologia de morte acidental em pessoas com idade de 65 anos ou mais. ${ }^{9}$ No Brasil, ocorreram 35.490 óbitos por causas externas entre pessoas com 60 anos ou mais, nos anos de 2005 e 2006. As quedas estão incluídas neste grupo como o evento que determinou $23 \%$ da morbimortalidade. ${ }^{10}$

A fratura de fêmur está entre as lesões traumáticas mais comuns na população idosa, podendo ocorrer na região proximal, distal ou ainda na diáfise femoral. ${ }^{11}$ Causada geralmente por traumas pequenos e não intencionais, como as quedas da própria altura, que ocorrem em função da senescência ou de fatores extrínsecos, representa importante causa de mortalidade e de incapacidade na população de 60 anos ou mais de idade. Enquanto uma mulher com fratura do fêmur proximal tem 1,5 vezes mais chance de morrer que uma sem fratura no período de dois anos, no sexo masculino essa probabilidade aumenta sete vezes mais. ${ }^{12}$ Vários estudos relataram que pacientes idosos com fratura do fêmur proximal alcançaram a capacidade prévia de realizar as tarefas diárias em apenas $17 \%$ das vezes após quatro meses e somente $43 \%$ readquiriram a capacidade comprometida. ${ }^{6,9,11}$

A literatura vem apontando que as doenças crônicas decorrentes da velhice podem agravar o quadro de saúde da pessoa idosa e impactar na qualidade de vida. Estudo realizado nos Estados Unidos entre 1991 e 2004 identificou a preexistência de doenças crônicas como diabetes mellitus $(25,5 \%)$ e hipertensão $(47,1 \%)$, em pacientes $(n=51)$ com histórico de fratura prévia decorrente de quedas. Do total, $64,7 \%$ tinham sua capacidade funcional comprometida: $35,3 \%$ parcialmente comprometida e $29,4 \%$ totalmente comprometida. ${ }^{13}$
Outro estudo, realizado na Holanda, com 7.046 pessoas de ambos os sexos (2778 homens, 4268 mulheres), para estudar o risco da fratura de quadril em indivíduos com baixa densidade mineral óssea (DMO), concluiu que o grupo de 70 anos ou mais de idade apresentou maior risco de fratura por osteoporose e predominou o sexo feminino. ${ }^{14} \mathrm{Na}$ Argentina, estudo realizado em 26 centros médicos de agosto de 2001 a julho de 2002, a fim de determinar a incidência de fratura de quadril na cidade de Rosário, concluiu que na população de 65 anos ou mais, a incidência foi 646 por 100.000 (847 para mulheres, 343 para homens); o sexo feminino sofreu 2,5 vezes mais quedas que o sexo masculino. $\mathrm{O}$ risco para indivíduos com idade inferior a 69 anos de idade foi o mesmo para ambos os sexos, porém a partir dos 70 anos aumenta exponencialmente para o sexo feminino. ${ }^{15} \mathrm{~A}$ fratura do colo femural do idoso tem como opções de tratamento a osteossíntese e as artroplastias parcial e total do quadril. ${ }^{16}$

As cirurgias de revisão são mais frequentes nos casos tratados com fixação, porém a taxa de mortalidade tardia (13 anos) é a mesma para todos esses tratamentos. ${ }^{17} \mathrm{O}$ tratamento da fratura tem por finalidade restaurar a anatomia óssea, a função do membro e reabilitar o paciente efetivamente, devolvendo sua funcionalidade para executar as atividades que realizava antes do evento.

Alguns estudos vêm demonstrando o impacto financeiro dos recursos médico-hospitalares utilizados no tratamento da fratura de fêmur para o sistema de saúde e para a sociedade. ${ }^{11,18} \mathrm{Na}$ Suécia, estudo de revisão realizado no ano de 2003 relatou que as doenças ósseas, apesar de serem comuns, não recebem muita atenção dos órgãos de saúde, mesmo com os prejuízos que causam para a sociedade. $O$ custo indireto do comprometimento músculo esquelético excede os custos diretos $(80 \%$ versus $20 \%) .{ }^{19} \mathrm{O}$ custo social e econômico da fratura de fêmur se eleva ainda mais pelo fato de que, após um período de tempo variável de internação, as taxas de mortalidade permanecem altas, os cuidados médicos permanecem intensivos e programas de reabilitação por longos períodos se tornam necessários. ${ }^{20}$ 
No Brasil, além do Sistema Único de Saúde (SUS) que garante assistência integral e gratuita para a totalidade da população, existe o setor privado de saúde, denominado Suplementar, que é composto pelas operadoras de planos individuais e coletivos, que contratam prestadores de serviços de saúde (hospitais, laboratórios, clínicas, médicos) para atenderem à carteira de beneficiários. $\mathrm{O}$ setor é regulado pela Agência Nacional de Saúde Suplementar (ANS), criada pela Lei n-9.961, de 28 de janeiro de 2000. A ANS regula as operadoras pela Lei no 9.656, de 03 de junho de 1998, que definiu os atributos essenciais dos planos de saúde, critérios para entrada, funcionamento e saída de operação de empresas no setor.

Os gastos com saúde (público e privado) estão no topo das agendas de todos os países do mundo. A princípio, em qualquer cenário econômico projetado, o crescimento do gasto com saúde per capita acompanha o próprio crescimento do PIB per capita, mantendo constante a participação do gasto com saúde em relação ao produto. No Brasil, em decorrência do envelhecimento da população, o gasto com saúde deverá crescer como proporção do PIB e deverá acarretar em 2050, um gasto total em saúde de $10,3 \%$ do PIB. ${ }^{21}$

A finalidade primária deste estudo foi estimar o custo direto do tratamento cirúrgico da fratura de fêmur decorrente de quedas em pessoas idosas e os gastos decorrentes da reabilitação no período de dois meses subsequentes ao tratamento cirúrgico da lesão. Os resultados poderão levar os financiadores da saúde a conhecerem dados inexistentes, fazer análise econômica e comparar medidas preventivas, terapêuticas e custos com fraturas para melhor alocar os escassos recursos financeiros.

\section{MÉTODOS}

\section{Tipo de pesquisa}

Estudo transversal, descritivo e retrospectivo com base documental sobre a estimativa dos custos diretos durante o período de internação, para o tratamento cirúrgico de fratura de fêmur e pósoperatório de até dois meses após a lesão.

\section{Universo e amostra}

O universo da pesquisa foi constituído por todos os casos de internação, decorrente de quedas, compreendidos no período de $01 \mathrm{de}$ janeiro de 2008 e 31 de junho de 2009, de beneficiários com 60 anos ou mais de idade, em cinco hospitais privados credenciados a um convênio da cidade de Brasília.

O critério de inclusão foi a autorização prévia de cirurgias ortopédicas de fratura de fêmur, cuja causa externa tenha sido queda, e sua associação com sexo e idade (60 anos ou mais) do paciente e todas as demais autorizações de procedimentos durante o período de dois meses pós-operatório (durante a internação e após a alta).

Os dados foram coletados no arquivo de processos administrativos e contas hospitalares de redução cirúrgica de fratura de fêmur. $O$ custo direto dos recursos médico-hospitalares utilizados no tratamento foram computados da seguinte forma:

- Tempo médio de internação (TMI): número de casos, dias de internação em UTI, dias de permanência; e

- Despesas hospitalares: honorários médicos, serviços auxiliares diagnósticos e terapêuticos (SADT), taxas hospitalares, materiais descartáveis e medicamentos, UTI, diárias, órteses e próteses e materiais especiais (OPME), anestesiologia e hemoderivados.

Os prontuários dos pacientes também foram analisados, para se identificar o histórico de doenças preexistentes, ou seja, os fatores intrínsecos que podem contribuir na ocorrência do evento queda (osteoporose, hipertensão arterial sistêmica, diabetes mellitus tipo II, quedas anteriores).

Os dados obtidos foram organizados utilizando-se planilha eletrônica $\left(\right.$ Excel $\left.^{\circledR}\right)$, sendo 
os resultados apresentados em quadros, tabelas e figuras, com distribuição de frequências e cálculos de medidas de tendência central e de dispersão gerados pelo programa (SPSS versão 13.0 para Windows $\left.{ }^{\circledR}\right)$.Os procedimentos foram selecionados conforme código escolhido pelo médico para redução cirúrgica e fixação da fratura. A descrição e o código do procedimento utilizado foram retirados da tabela da Associação Médica Brasileira (AMB 92): As faixas etárias foram distribuídas da seguinte forma: $60-69,70-79$ e 80 ou mais.

A coleta de dados seguiu o estabelecido no termo de concordância da instituição e foram observados os preceitos estabelecidos na Resolução n 196/96, do Conselho Nacional de Saúde. A pesquisa foi aprovada pelo Comitê de Ética da Universidade Católica de Brasília sob número de registro CEP/UCB 067/2009.

Pela ausência de pesquisas que identifiquem os custos da fratura de fêmur decorrente de queda em idosos, na cidade de Brasília, o trabalho ficará restrito às informações de um único convênio da saúde suplementar que podem refletir ou não os custos de outros convênios. Diante das limitações apresentadas pelo número pequeno de pacientes que compõem a amostra, e pelo fato de a saúde suplementar não ter os dados investigados disponíveis no Sistema de Informações de Saúde (DATASUS), o estudo pode ser considerado um experimento de fase inicial. $O$ fato de o estudo ser retrospectivo acarreta potencial perda de dados, que poderiam ser coletados em estudos prospectivos.

\section{RESULTADOS}

Houve predomínio da fratura de fêmur proximal $78,6 \%(\mathrm{n}=11)$ para o sexo feminino. A tabela 1 apresenta a relação entre o sexo e o local da queda que resultou a lesão, onde $n=9(42,8)$ corresponde a quedas da cama, jogando futebol, no quintal e na cozinha).

Tabela 1 - Relação entre o sexo e local da queda das fraturas de fêmur no setor privado de saúde. Brasília, DF, 2008-2009.

\begin{tabular}{|c|c|c|c|}
\hline \multirow[b]{2}{*}{ Local } & \multicolumn{3}{|c|}{ Média e frequência percentual } \\
\hline & Feminino & Masculino & Total \\
\hline Banheiro & $\mathrm{n}=5(31,3)$ & $\mathrm{n}=3(60)$ & $\mathrm{n}=8(38,0)$ \\
\hline Rua & $\mathrm{n}=4(25,0)$ & $\mathrm{n}=0(00)$ & $\mathrm{n}=4(19,0)$ \\
\hline Outros & $\mathrm{n}=7(43,8)$ & $\mathrm{n}=2(40)$ & $\mathrm{n}=9(42,8)$ \\
\hline
\end{tabular}

Os fatores de risco intrínsecos relacionados às doenças crônicas pré-queda que foram identificados nos pacientes integrantes da amostra são: reumatológica/ortopédica $(100 \%) \mathrm{n}=21$, cardiovascular $(85,7 \%) \mathrm{n}=18$ e endócrinometabólica $(42,9 \%) n=9$.

As quedas resultaram em 16 fraturas de fêmur, sendo $(n=14)$ proximais (colo do fêmur, coxofemural, trans-trocanteriana cominutiva) e $(\mathrm{n}=2)$ distais (patela femural, joelho-fêmur). A primeira queda ocorreu para $80,9 \%$ dos idosos e $19 \%$ relataram pelo menos uma queda anterior, tendo como resultado outro tipo de lesão (crânio, punho, tíbia, joelho e ombro). Houve apenas um caso de cirurgia de revisão de fratura fêmur (quadril) relacionada à soltura da prótese primária em decorrência de osteomelite.

A taxa de mortalidade do grupo foi $19 \%$ e os óbitos ocorreram no período pósoperatório intra-hospitalar $(\mathrm{n}=3)$ ou até dois meses após a alta hospitalar $(n=1)$. Não se pode afirmar que a fratura foi a causa direta do óbito, mas que contribuiu indiretamente para o evento. 
A tabela 2 apresenta o tempo médio de internação para o tratamento cirúrgico da fratura de fêmur. A faixa etária de 60-69 demandou maior tempo de recuperação intra-hospitalar. O resultado se inverte para a permanência em Unidade de Terapia Intensiva, que foi maior para o grupo de 80 ou mais.

Tabela 2 - Tempo médio de internação para o tratamento cirúrgico de fratura de fêmur, por faixa etária no setor privado de saúde. Brasília, DF, 2008-2009.

\begin{tabular}{lcccc}
\hline & \multicolumn{3}{c}{ Faixa Etária (anos) } & Total \\
\cline { 2 - 4 } \multicolumn{1}{c}{ Variáveis } & $60-69$ & $70-79$ & 80 ou mais & 16 \\
\hline $\mathrm{N}^{\circ}$ de casos(n) & 5 & 3 & 8 & $7,1 \pm 13,6$ \\
& & $2,5 \pm 0,577$ & $5,3 \pm 5,7$ & $2,7 \pm 2,3$ \\
Tempo médio de & $13,4 \pm 23,8$ & $2,2 \pm 1,2$ & $3,6 \pm 2,9$ & \\
$\begin{array}{l}\text { internação (dias) } \\
\text { Tempo médio de }\end{array}$ & $1,8 \pm 1,9$ & & & \\
UTI (dias) & & &
\end{tabular}

Os custos diretos dos recursos médicohospitalares utilizados na redução cirúrgica das fraturas de fêmur no período de hospitalização ficaram entre $\mathrm{R} \$ 8.293,55$ e $\mathrm{R} \$ 139.837,50$, e totalizaram $\mathrm{R} \$ 626.572,06$. O custo médio foi maior na faixa etária de 70-79 anos de idade e correspondeu a $\mathrm{R} \$ 10.165,00$. O desvio padrão dos gastos com as próteses ( $\mathrm{R} \$ 21.570,22)$ foi significativo para a faixa etária 60-69 anos de idade, se comparada aos grupos de pessoas mais idosas. O custo direto da hospitalização desembolsado pelo convênio à rede hospitalar privada foi em média o valor de $\mathrm{R} \$ 39.160,74$ para todas as fraturas. Os recursos médico-hospitalares mais dispendiosos foram: próteses e materiais de alto custo $(49,6 \%)$, materiais descartáveis e medicamentos (14,4\%), honorários (12,0\%) e taxas hospitalares $(9,5 \%)$ (tabela 3$)$.

Tabela 3 - Custo direto dos recursos médico-hospitalares da cirurgia de fratura de fêmur no setor privado de saúde. Brasília, DF, 2008-2009.

\begin{tabular}{lcc}
\hline Recurso $(\mathrm{R} \$)$ & Valor médio & Percentual \\
\hline Diárias & $2.557,83 \pm 3.486,05$ & $6,5 \%$ \\
Honorários & $4.721,14 \pm 2.809,00$ & $12,0 \%$ \\
Taxas & $3.714,70 \pm 2.417,62$ & $9,5 \%$ \\
Materiais/ & $5.638,31 \pm 6.395,76$ & $14,4 \%$ \\
Medicamentos & & \\
OPME & $19.401,11 \pm 14.119,45$ & $49,6 \%$ \\
Outros & $3.127,65 \pm 2.863,64$ & $8,0 \%$ \\
\hline Total $(\mathrm{R} \$)$ & $39.160,74 \pm 32.091,52$ & $100 \%$ \\
\hline
\end{tabular}


A figura 1 representa a despesa da reabilitação em até dois meses da alta hospitalar do paciente. Os recursos médico-hospitalares em bome care, fisioterapia e serviços auxiliares, diagnósticos e terapêuticos representaram $5,7 \%$ do custo total do tratamento cirúrgico.

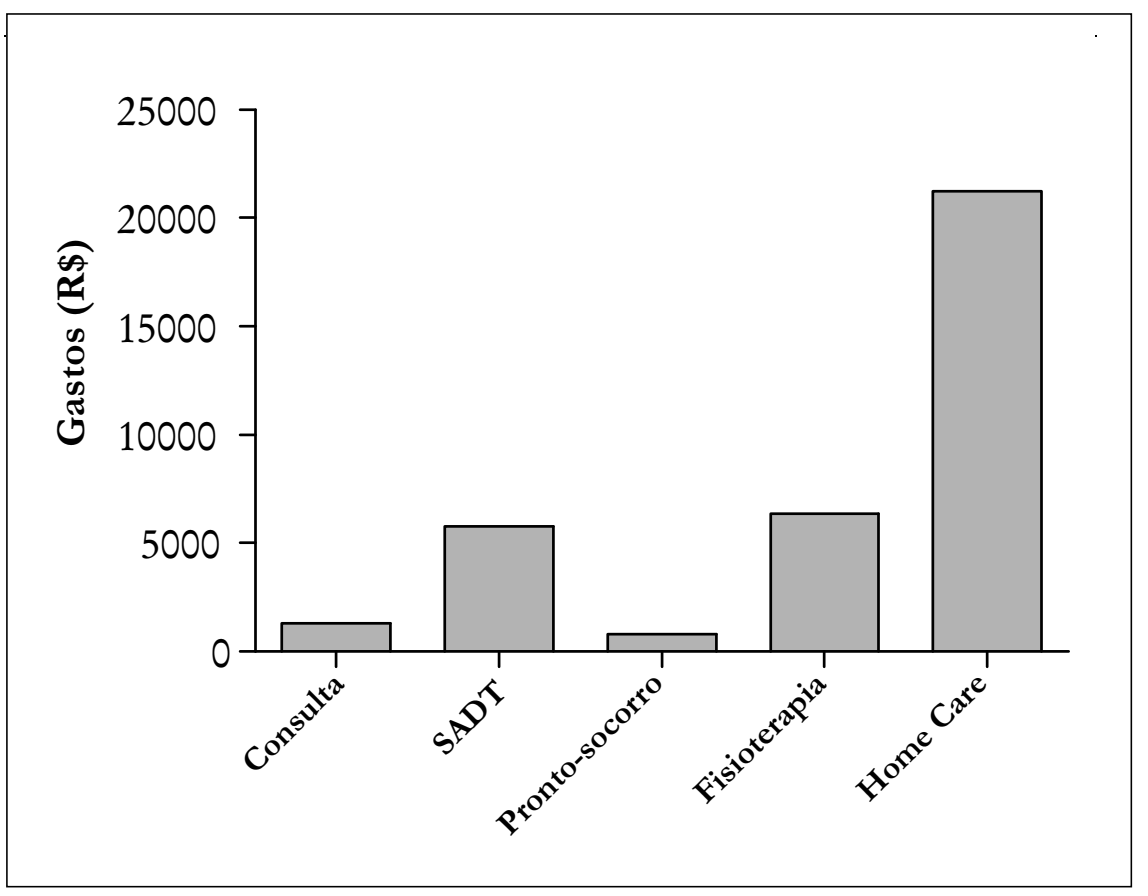

Figura 1 - Custo direto recursos médico-hospitalares pós-operatório até 2 meses do tratamento cirúrgico fratura fêmur. Setor privado de saúde, Brasília, DF, 2008-2009.

\section{DISCUSSÃO}

O estudo confirmou o que a literatura já vem apontando: a vulnerabilidade do sexo feminino diante do evento queda, cuja lesão principal foi fratura de fêmur proximal (quadril). Estudo de revisão realizado no Brasil, entre o período de 1997 e 2007, nas bases de dados eletrônicas SCiELO, Lilacs e Medline, confirmou que 5\% das quedas resultam em lesões graves e são responsáveis por mais de 200 mil hospitalizações por fratura de quadril a cada ano. ${ }^{22}$ Existem dois componentes responsáveis pela resistência óssea: a densidade e a qualidade óssea. Tais componentes começam a declinar no sexo feminino após a menopausa, devido à redução dos estrógenos, e algumas mulheres passam a perder massa óssea acima de $1 \%$ ao ano, sendo que algumas chegam a perder $5 \%$ e, no final de cinco anos, estão com perda superior a $25 \%$, caracterizando a osteoporose pós-menopausa. ${ }^{23}$
A literatura brasileira e internacional aponta a osteoporose como um dos fatores de risco mais importantes quando associada à ocorrência de fratura de quadril na população de 60 anos ou mais de idade. ${ }^{16,25,26} \mathrm{~A}$ maior incidência da fratura de quadril em mulheres pode estar relacionada ao fato de iniciar o processo de declínio de massa óssea antes do homem. O presente estudo revelou que todos os pacientes possuíam histórico de doenças reumatológica/ortopédica (osteopenia, osteoartrose, osteoporose), o que confirma os dados alarmantes da prevalência desse grupo de doença em pessoas idosas.

Estudo sobre a prevalência da fratura osteoporótica de fêmur em idosos, realizado no ano de 2005 , ressaltou que a maioria dos pacientes que sofreram fratura de fêmur não iniciou tratamento específico, pois apenas de $6 \%$ a $10 \%$ são orientados para tal no momento da alta. ${ }^{27}$ Outra investigação realizada no mesmo ano, que 
corrobora a ausência dessa conduta no presente estudo, foi a constatação de que $40 \%$ dos pacientes internados por fratura de fêmur não relacionavam a lesão à osteoporose. ${ }^{28}$ Doenças crônicas, como diabetes mellitus tipo II, hipertensão arterial sistêmica e doenças cardiovasculares também podem aumentar o risco de quedas em idosos. ${ }^{29} \mathrm{~A}$ incidência desses fatores intrínsecos nos indíviduos selecionados na amostra revelou que a frequência de hipertensão no sexo feminino foi $93,8 \%$ para $60 \%$ no sexo masculino. Estudo realizado no Brasil no ano de 2000 identificou em pacientes idosos internados com fratura de quadril perfil epidemiológico semelhante ao dos indivíduos da presente investigação. As principais doenças preexistentes antes da lesão foram: hipertensão arterial (68\%), diabetes (34\%) e osteoporose (19\%). ${ }^{30}$

Outra pesquisa tipo caso-controle realizada no Brasil, no ano de 2004, com 205 pacientes com 60 anos ou mais, admitidos no Serviço de Ortopedia e Traumatologia da Faculdade de Medicina de Catanduva (SP), vítimas de fraturas por queda, concluiu que existe correlação entre o uso de medicamento e o evento. O percentual de idosos que faziam uso de algum medicamento nas 24 horas que antecederam o trauma foi $72,5 \%$, e os principais medicamentos identificados neste grupo foram o ácido acetilsalicílico (AAS), amiodarona (antiarrítmico), atenolol e captopril (anti-hipertensivo). A hipotensão postural induzida pelo medicamento pode ter sido a causa da queda. ${ }^{31}$ Em média, $47,6 \%$ do grupo estudado possuíam relato, em prontuário, sobre uso contínuo de anti-hipertensivo, porém a ausência de registros sobre a queda nos prontuários impediu a associação desses dados com o uso prévio da medicação. A taxa de mortalidade encontrada na amostra (19\%) corresponde à análise da variável até dois meses após a cirurgia, sendo que a literatura apresenta taxa semelhante em um ano de avaliação do paciente.

Pinheiro et. al. ${ }^{32}$ (2009) realizaram estudo de relacionamento probabilístico no Brasil sobre a mortalidade em um ano após a redução cirúrgica de fratura de quadril em idosos de acordo com o período pós-operatório, na cidade do Rio de
Janeiro, no período de $1^{\circ}$ de janeiro e 31 de dezembro de 1995, reticulado com os dados do Sistema Brasileiro de Informações sobre Mortalidade (SIM), relativos ao mesmo período e mesma região, ajustados por três períodos de tempo: 1-30, 31-90 e 91-365 dias. Encontraram uma taxa de mortalidade de $21,5 \%$ em um ano, constatando que a morte ocorreu no período de internação ou após alta hospitalar. No mesmo estudo, identificou-se ainda que as três principais causas básicas do óbito foram os eventos cardiovasculares, quedas e infecções. Outro estudo realizado no Brasil, no ano de 2006, em 190 pacientes internados com fratura da extremidade proximal do fêmur, no Hospital dos Servidores do Estado de Minas Gerais, encontrou taxa de mortalidade semelhante ao desta pesquisa $(19,5 \%)$ em pacientes acompanhados até um ano pósfratura. ${ }^{33}$

No presente estudo, os custos diretos dos recursos médico-hospitalares utilizados na redução cirúrgica das fraturas de fêmur no período de hospitalização totalizaram $R \$ 626.572,06$. O custo médio para todas as fraturas $(n=16)$ foi $R \$$ $39.160,74 \pm \mathrm{R} \$ 32.091,52$; já para a faixa etária de 60-69 anos de idade $(n=5)$ foi maior: $R \$$ $50.824,98 \pm R \$ 42.046,62$.

É provável que essa diferença prevaleceu em decorrência da cirurgia de revisão de artroplastia total de quadril existente no grupo. A revisão da prótese total de quadril pode ter o dobro do custo da primária, em razão das complicações associadas a fixação do material e qualidade óssea do paciente. ${ }^{34} \mathrm{O}$ custo direto da fratura osteoporótica de fêmur no período de hospitalização, valorado pelo setor de saúde suplementar brasileiro no ano de 2005, foi estimado em $\mathrm{R} \$ 24.051,17 .{ }^{18}$ Embora este estudo apresente um número de indivíduos inferior, existe a percepção de que o custo com os recursos médico-hospitalares utilizados no tratamento cirúrgico das fraturas de fêmur foi significativo em ambos os casos.

As despesas não terminam com a alta hospitalar e o custo da assistência permanece significativo até a reabilitação do paciente. A análise dos recursos médico-hospitalares 
utilizados no período de até dois meses após a cirurgia correspondeu a $5,7 \%$ dos recursos utilizados no período intra-hospitalar. As despesas com home care, fisioterapia e SADT consumiram juntos cerca de $94 \%$ dos recursos médicohospitalares empregados na assistência pósoperatória.

Estudo de revisão realizado de 1980 a 2003, considerando 18 países da América Latina, para investigar a presença de osteopenia no colo do fêmur em mulheres, identificou que o custo direto do tratamento cirúrgico para fratura de quadril era de $\$ 5.500$. Este valor atualizado ( $\mathrm{R} \$ 1,739$, cotação em 27/10/2009) significa o montante de $\mathrm{R} \$ 9.564,50$. O mesmo estudo relata o custo do tratamento de um protocolo básico que incluiu: exame de densiometria óssea, cálcio, alendronato e calcitonina (\$187), o que corresponde a ( $\mathrm{R} \$$ $325,19) \cdot{ }^{26}$

Araújo et al. ${ }^{18}$ analisaram o custo-efetividade do ácido zoledrônico (dose anual) versus residronato (dose mensal) e concluíram que para uma boa resposta de qualquer fármaco, é imprescindível a aderência do paciente ao tratamento. Os resultados da análise econômica demonstraram que o ácido zoledrônico, sendo uma opção com maior aderência, revelou ser mais custo-efetivo por fratura proximal de fêmur evitada em cinco anos. Os resultados do presente estudo demonstram a importância do tratamento e controle da osteoporose na redução dos riscos que desencadeiam a fratura no idoso, além de garantir menores gastos com a assistência.

Poucos estudos sobre custos diretos dos recursos médico-hospitalares utilizados no tratamento da fratura de fêmur decorrente de quedas no sistema de saúde privado do Brasil foram localizados.

$\mathrm{Na}$ realização da presente pesquisa, é digno de destaque o preenchimento dos prontuários. Constatou-se ausência de informações sobre a queda como causa básica da internação; sobre a preexistência de doenças crônicas; sobre o uso contínuo de medicamentos (antes da queda); e não menos importante, sobre a qualidade óssea do paciente. Em relação a esse último aspecto, ressalta-se que em nenhum dos prontuários verificados houve referência à realização de exame de densitometria óssea e ao tratamento da osteoporose. Abreviações e nomenclaturas inventadas para reduzir o texto e ausência de padronização no relato das condições do paciente no momento da baixa hospitalar foram aspectos também encontrados e que acabam por trazer limitações à análise aqui proposta.

\section{CONCLUSÃO}

A presente pesquisa constatou que a fratura de quadril é um grave problema para a gestão financeira do setor privado de saúde e representa uma iniciativa que visa a concientizar os vários atores do sistema para a importância do custo na tomada de decisão. A pessoa idosa merece destaque neste cenário, por ser o principal ator da tríade: sistema (forma de remuneração dos serviços de saúde), assistência (tecnologia em crescente inflação) e resultado (custo-benefício) do tratamento. A incapacidade funcional e a alta taxa de mortalidade pós-cirúrgica revelam que estudos de análise econômica que relatem o custoefetividade do tratamento das doenças crônicas, associados a um estilo de vida saudável, merecem prioridade nas diretrizes e políticas da Agência Nacional de Saúde.

A Política Nacional da Saúde do Idoso (Portaria $\left.n^{\circ} 1395-G M / 1999\right)$ propugna a promoção do envelhecimento saudável e ativo, que tem como fatores determinantes a nutrição, a atividade física, a prevenção da obsesidade, do tabagismo e do consumo excessivo de àlcool, bem como outros fatores socioeconômicos. Entre as várias ações que podem determinar as mudanças necessárias rumo ao envelhecimento ativo, está a importância da sensibilização dos profissionais de saúde quanto a sua prática e o entendimento de que o paciente precisa estar mais informado e consciente dos problemas relacionados com sua doença para a promoção autocuidado.

O desenvolvimento de pesquisas nacionais que aperfeiçoem a matéria-prima usada na fabricação 
de implantes cirúrgicos e que apresentem relação custo-eficácia pós-cirúrgica podem estimular a competitividade nacional e reduzir o valor das próteses utilizadas nas artroplastias.

O presente estudo não incluiu a análise de custo não-médico hospitalar direto e indireto (perdas de produtividade, lazer, transporte do idoso e de familiares) e, ao longo da pesquisa

\section{REFERÊNCIAS}

1. Instituto Brasileiro de Geografia e Estatística (IBGE). População brasileira envelhece em rítmo acelerado. Brasília 2009 [Acesso 10 Set 2009].Disponível em: URL: http:// www.ibge.gov.br/home/presidencia/noticias/ noticia_visualiza.php? id_noticia $=1272 \&$ id_pagina.

2. Bureau of the Census. Population projections program: population division. Washington 2007 [Acesso 10 out 2007].Disponível em: URL: http://www.census.gov/population/www/ projections/popproj.html.

3. Camargos MCS, Perpétuo IHO, Machado CJ. Expectativa de vida com incapacidade funcional em idosos em São Paulo. Rev Panam Salud Publica $2005 ; 17(5 / 6)$ : 379-86.

4. Parahyba MI, Veras R. Diferenciais no declínio funcional em mobilidade física entre idosos no Brasil. Ciênc Saúde Coletiva 2008; 13(4): 125764.

5. Fabrício SCC, Rodrigues RAP, Costa Júnior ML. Quedas acidentais em idosos institucionalizados. Acta Paul Enf 2002; 15(3): 51-59.

6. Paixão Junior CM, Heckmann M. Distúrbios da postura, marcha e quedas. In: Freitas EV, et al (Orgs). Tratado de Geriatria e Gerontologia. Rio de Janeiro: Guanabara Koogan; 2002.

7. Moura RN ,et al. Quedas em idosos: fatores de risco associados. Gerontologia 1999; 7(2): 15-21.

8. Ribeiro,et al. A Influência das quedas na qualidade de vida de idosos. Ciênc Saúde Coletiva 2008; 13(4): 1265-1273.

9. Fuller GF. Falls in the elderly. Am Family Physician 2000; 61(21): 2159-68.

10. Sistema de Informações sobre Mortalidade ( SIM). Disponível em: URL: http:// w3.datasus.gov.br/datasus/datasus.php. bibliográfica, foi constatada a importância desses dados no detalhamento de custos para posteriores análises econômicas.

\section{AGRADECIMENTOS}

Agradecemos a todos que direta e indiretamente contribuíram para a conclusão do presente estudo.
11. Muniz CF, Arnaut AC, Yoshida M, Trelha CS. Caracterização dos idosos com fratura de fêmur proximal atendidos em hospital escola público. Rev Espaço Saúde Londrina 2000;8(2): 33-38.

12. Fransen $M$,et al. Excess mortality or institucionalization after hip fracture: men are at greater risk than women. J Am Geriatr Soc 2002; 50: 685-90.

13. Mizrahi EH. The impact of previus strokes on the rehabilitation of elderly patients sustaining a hip fracture. Arch Phys Med Rehabil 2007; 88: $1136-9$.

14. Chris ED. Hip Fracture Prediction in Elderly Men and Women : validation in the rotterdam study. J. Bone Mineral Research 1998; 13(10): 1587-93.

15. Morosano M, Masoni A, Sánchez A. Incidence of hip fractures in the city of Rosario. Osteopor Int 2005; 16(11):1339-44.

16. Sakaki $\mathrm{MH}$,et al. Estudo da mortalidade na fratura do fêmur proximal em idosos. Acta Ortop Bras 2004; 12(4): 242-249.

17. Ravikumar KJ, Marsh G. Internal fixation versus hemiarthroplasty versus total hip arthroplasty displaced subcapital fractures of femur-13 year results of a prospective randomised study. Injury 2000; 31:793-797.

18. Araújo DV ,et al. Análise do custo-efetividade do ácido zoledrônico na prevenção da fratura osteoporótica proximal de fêmur no cenário do sistema suplementar de saúde brasileiro. Rev. Bras Geriatr Gerontol 2008; 11(3):357-68.

19. Akesson $\mathrm{K}$. Bone and joint diseases around the world : Sweden: a brief update on burden and priority. J Rheumatol 2003; 67: 21-5.

20. Hannan EL ,et al. Mortality and locomotion 6 months after hospitalization for hip fracture: risk factors and risk-adjusted hospital outcomes. JAMA 2001; 285: 2736-42. 
21. Associação Brasileira de Empresas de Pesquisa (ABEP). Serviços de saúde, gastos e envelhecimento da população brasileira. 2000 [Acesso 27 Out 2000].Disponível em:URL: http://abep.org.br/usuario/ GerenciaNavegacao.php?busca $=$ Pesquisar\&caderno $\mathrm{id}=$ 305\&listaCadernos $=\&$ palavraChave $=$ kilsztajn .

22. Messias M, Neves RF. A influência de fatores comportamentais e ambientais domésticos nas quedas em idosos. Rev Bras Geriatr Gerontol 2009; 12(2): 275-282.

23. Russo LAT. Osteoporose pós-menopausa: opções terapêuticas. Arq bras Endocrinol Metab 2001; 45(4): 401-6.

24. Schwartz AV ,et al. International variation in the incidence of hip fractures: cross-national project on osteoporosis dor the World health. Osteoporos Int 1999; 9(3): 242-253.

25. Rocha FAC, Ribeiro AR. Low incidence of hip fractures in equatorial area. Osteoporos Int 2003; 14: 496-99.

26. Morales-Torres J, Gutiérrez-Urenã S. The burden of osteoporosis in Latin America. Osteoporos Int 2004; (15): 625-632.

27. Farias MLF. Fratura Osteoporótica de Fêmur : um desafio para os sistemas de saúde e a sociedade em geral. Arq Bras Endocrinol Metab 2005; 49(6): 865-66.
28. Gardner MJ ,et al. Interventions to improve osteoporosis treatment following hip fracture. J Bone Joint Surg 2005; 87: 3-7.

29. Lawlor DA, Patel R, Ebrahim S. Association between falls in elderly women and crhonic diseases and drugs in use. BMJ 2003; 327: 1-6.

30. Garcia R, Leme MD, Garcez-Leme LE. Evolution of Brazilian elderly with hip fracture secondary to a fall. Clinics 2006; 61(6): 539-544.

31. Hamra A, Ribeiro MB, Miguel OF. Correlação entre fratura por queda em idosos e uso prévio de medicamentos. Acta Ortopédica 2007; 15(3): 143-5.

32. Pinheiro MM, et al. Clinical risk factors for osteoporotic fractures in brazilian women and men: the Brazilian Osteoporosis Study (BRAZOS). Osteoporos Int 2009; 20: 399 408.

33. Cunha U, Veado MAC. Fratura da extremidade proximal do fêmur em idosos: independência funcional e mortalidade em um ano. Rev Bras Ortop 2006; 41(6): 195-9.

34. Rodrigues CVM. Próteses totais de quadril: diagnóstico do panorama nacional e elaboração de diretrizes para políticas públicas. Rio de Janeiro. Tese [Doutorado] — Universidade Federal do Rio de Janeiro; 2001. 
